\title{
Limits for Recombination in a Low Energy Loss Organic Heterojunction
}

\author{
S. Matthew Menke, ${ }^{\dagger}$ Aditya Sadhanala, ${ }^{\dagger}$ Mark Nikolka, ${ }^{\dagger}$ Niva A. Ran, ${ }^{\ddagger}$ Mahesh Kumar Ravva, ${ }^{\S}$
} Safwat Abdel-Azeim, ${ }^{\S}$ Hannah L. Stern, ${ }^{\dagger}$ Ming Wang, ${ }^{\ddagger}$ Henning Sirringhaus, ${ }^{\dagger}$ Thuc-Quyen Nguyen, ${ }^{\ddagger}$ Jean-Luc Brédas, ${ }^{\S}$ Guillermo C. Bazan, ${ }^{\ddagger}$ and Richard H. Friend ${ }^{*}{ }^{\dagger}$

\author{
${ }^{\dagger}$ Department of Physics, Cavendish Laboratory, University of Cambridge, J.J. Thompson Avenue, Cambridge CB3 0HE, United \\ Kingdom \\ ${ }^{\ddagger}$ Center for Polymers and Organic Solids, University of California, Santa Barbara, California 93106, United States \\ ${ }^{\S}$ Solar \& Photovoltaics Engineering Research Center, Division of Physical Science and Engineering, King Abdullah University of \\ Science and Technology, Thuwal 23955-6900, Saudi Arabia
}

\section{Supporting Information}

\begin{abstract}
Donor-acceptor organic solar cells often show high quantum yields for charge collection, but relatively low open-circuit voltages $\left(V_{\mathrm{OC}}\right)$ limit power conversion efficiencies to around $12 \%$. We report here the behavior of a system, PIPCP:PC ${ }_{61} \mathrm{BM}$, that exhibits very low electronic disorder (Urbach energy less than $27 \mathrm{meV}$ ), very high carrier mobilities in the blend (field-effect mobility for holes $>10^{-2} \mathrm{~cm}^{2} \mathrm{~V}^{-1} \mathrm{~s}^{-1}$ ), and a very low driving energy for

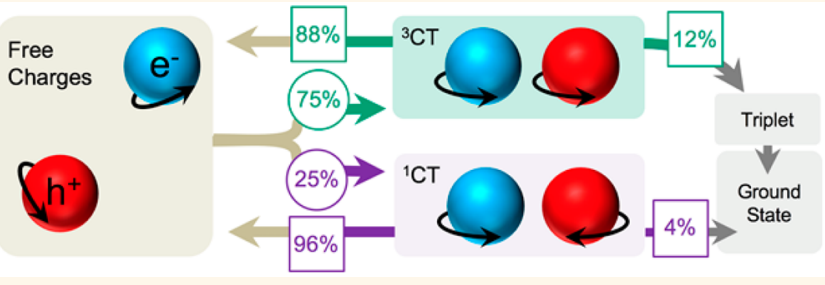
initial charge separation $(50 \mathrm{meV})$. These characteristics should give excellent performance, and indeed, the $V_{\mathrm{OC}}$ is high relative to the donor energy gap. However, we find the overall performance is limited by recombination, with formation of lower-lying triplet excitons on the donor accounting for $90 \%$ of the recombination. We find this is a bimolecular process that happens on time scales as short as 100 ps. Thus, although the absence of disorder and the associated high carrier mobility speeds up charge diffusion and extraction at the electrodes, which we measure as early as 1 ns, this also speeds up the recombination channel, giving overall a modest quantum yield of around $60 \%$. We discuss strategies to remove the triplet exciton recombination channel.
\end{abstract}

KEYWORDS: organic solar cell, energy loss, high mobility, charge recombination, charge transfer states

$\mathrm{F}$ or organic solar cells (OSCs), dissociation of molecular excitons occurs at the interface between an electrondonating species and an electron-accepting species. ${ }^{1,2}$ As a consequence of this design, significant energetic losses are generally incurred when the resulting intermolecular charge transfer (CT) states relax toward their energetic minima and undergo nonradiative decay processes. ${ }^{3}$ These losses are evident in the large difference between the optical energy gap $\left(E_{\text {opt }}\right)$ and the open-circuit voltage $\left(V_{\mathrm{OC}}\right)$, with most OSCs showing an energy loss $\left(E_{\text {loss }}=E_{\text {opt }}-e V_{\mathrm{OC}}\right)$ of $>1 \mathrm{eV}{ }^{4}$ Strategies to reduce energy loss are imperative to move toward the radiative limit for energy loss of approximately $0.3 \mathrm{eV}$ established by Shockley and Queisser and achieve power conversion efficiencies $\left(\eta_{\mathrm{PCE}}\right)$ greater than $12 \%{ }^{5}$

Energy loss in OSCs can be separated into two categoriesthat which is incurred during the formation of charges or CT states and that which is incurred due to the recombination of charges or CT states. ${ }^{6}$ The energy of the charge transfer state $\left(E_{\mathrm{CT}}\right)$ in relation to $E_{\mathrm{opt}}$ and $e V_{\mathrm{OC}}$ signals through which channel the majority of energy loss is occurring (Figure 1a). If $E_{\mathrm{CT}}$ is closer to $E_{\mathrm{opy}}$ most of the energy loss is due to recombination. Conversely, if $E_{\mathrm{CT}}$ is closer to $e V_{\mathrm{OC}}$, most of the energy loss occurs during the formation of CT states. In order to increase $V_{\mathrm{OC}}$, one should seek to simultaneously increase the value of $E_{\mathrm{CT}}$ while limiting the pathways for recombination.

Previous reports of OSCs with small energy loss exhibit a promising trend. ${ }^{7-9} \mathrm{Li}$ et al. have demonstrated OSCs with low energy loss $\left(E_{\text {loss }}<0.6 \mathrm{eV}\right)$ for a series of diketopyrrolopyrrole (DPP) copolymers yet report a systematic reduction in maximum external quantum efficiency $\left(\eta_{\mathrm{EQE}}\right)$ from $\eta_{\mathrm{EQE}}=$ $52 \%$ for $E_{\text {loss }}=0.58 \mathrm{eV}$ to $\eta_{\mathrm{EQE}}=5 \%$ for $E_{\text {loss }}=0.48 \mathrm{eV}{ }^{4}$ Kawashima et al. have reported $\eta_{\mathrm{PCE}}>8 \%$ from a blend of a quaterthiophene copolymer and $\mathrm{PC}_{71} \mathrm{BM}^{10}$ These devices exhibit $\eta_{\mathrm{EQE}} \sim 60 \%$ and $E_{\text {loss }}=0.56 \mathrm{eV}$. In both of these works, a small energy difference exists between donor polymer and fullerene acceptor electron affinities, likely indicating a high $E_{\mathrm{CT}}$ relative to $E_{\mathrm{opt}}$.

Received: September 14, 2016

Accepted: October 25, 2016

Published: November 3, 2016 
a.

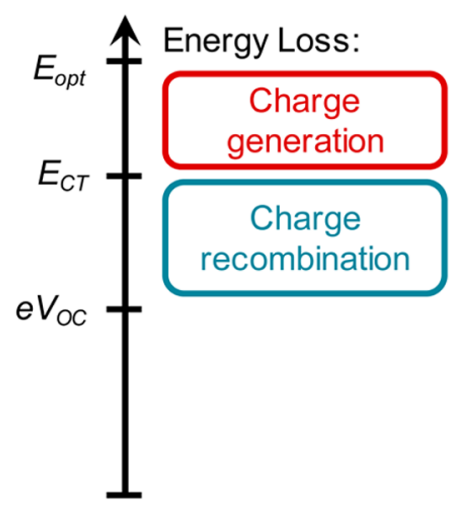

c. PIPCP

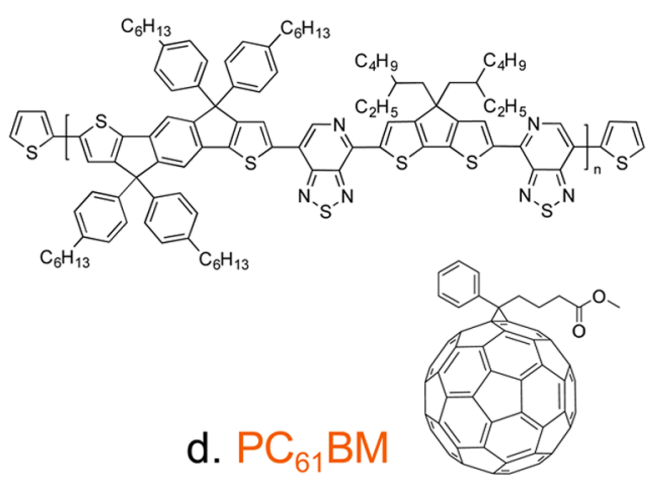

b.
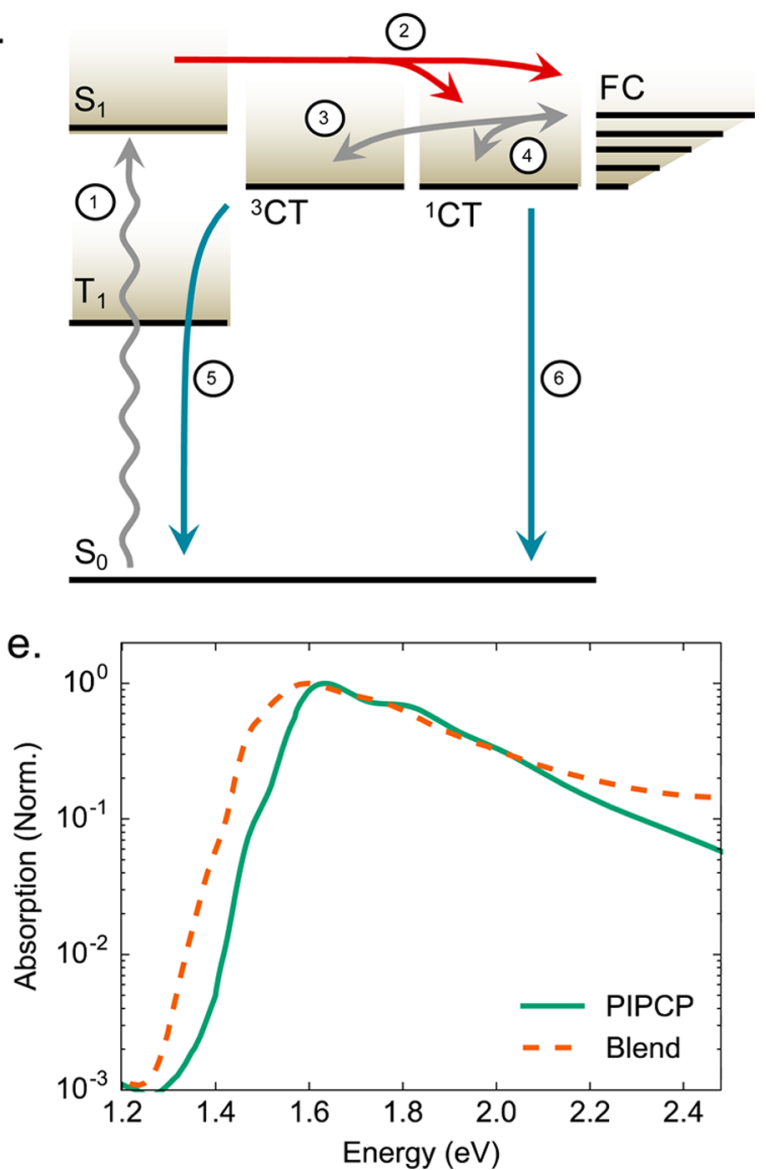

Figure 1. Energy loss in a low electronic disorder organic heterojunction. (a) Energy loss is defined as the energy difference between the optical energy gap $\left(E_{\mathrm{opt}}\right)$ and the energy of the open-circuit voltage $\left(e V_{\mathrm{OC}}\right)$. Loss incurred during charge generation is captured by the difference between $E_{\mathrm{opt}}$ and the energy of the charge transfer state $\left(E_{\mathrm{CT}}\right)$, whereas loss incurred due to charge recombination is captured as the difference between $E_{\mathrm{CT}}$ and $e V_{\mathrm{OC}}$. (b) Charge generation and recombination pathways in an organic heterojunction: (1) photon absorption to form singlet excitons, (2) charge transfer to form singlet, geminate CT states and free charges (FC), $(3,4)$ bimolecular recombination of FC to form nongeminate singlet and triplet CT states, (5) charge decay via energy transfer to triplet excitons, and (6) charge decay via direction recombination to the ground state. (c) Molecular structure of PIPCP and (d) PC ${ }_{61}$ BM. (e) Steady-state absorption spectra for PIPCP and a blend of PIPCP and $\mathrm{PC}_{61} \mathrm{BM}$ measured by photothermal deflection spectroscopy.

As the $E_{\mathrm{CT}}$ is increased, charge recombination becomes the dominant energy loss channel. While charge recombination energy loss is routinely measured and reported in the field, it has remained unclear which mechanisms are ultimately responsible. $^{11,12}$ When electrons and holes recombine with their spins uncorrelated, CT states with singlet $\left({ }^{1} \mathrm{CT}\right)$ and triplet $\left({ }^{3} \mathrm{CT}\right)$ character are formed (Figure $\left.1 \mathrm{~b}\right)$. Consequently, the nonradiative decay mechanisms for ${ }^{1} \mathrm{CT}$ and ${ }^{3} \mathrm{CT}$ states will determine the degree of charge-recombination-induced energy loss. For ${ }^{3} \mathrm{CT}$ states, energy transfer to lower-lying triplet excitons $\left(\mathrm{T}_{1}\right)$, usually on the donor material, has been shown to be an important decay mechanism. ${ }^{13}$ Recent studies on systems with low $\mathrm{T}_{1}$ energies have shown that this process happens on nanosecond to microsecond time scales at the expected 3:1 ratio derived from spin statistics. ${ }^{14}$ For ${ }^{1} \mathrm{CT}$ states, the picture is less clear. However, as no change in spin is required to decay to the ground state $\left(S_{0}\right)$, direct conversion from ${ }^{1} \mathrm{CT}$ to $S_{0}$ is the most likely decay mechanism, with smaller ${ }^{1} \mathrm{CT}$ energies increasing the rate of nonradiative recombination. ${ }^{15}$

Beyond energy loss, future OSCs will require increases in fill factor (FF) in order to achieve $\eta_{\mathrm{PCE}}>15 \%$. Several groups have performed device simulations which show that balanced charge mobilities $>10^{-2} \mathrm{~cm}^{2} \mathrm{~V}^{-1} \mathrm{~s}^{-1}$ are required to achieve $\mathrm{FF} \sim$
0.8. ${ }^{16-18}$ High mobilities can be achieved for fullerene acceptor phases in most state-of-the-art OSCs, but the mobility for polymeric donor phases has routinely lagged. While high charge mobilities improve charge extraction, they may, by the same token, increase encounters between opposite charges and thus increase the opportunity for charge recombination. Establishing a clear picture for charge recombination and decay for a high mobility, low energy loss OSC heterojunction becomes of tantamount importance.

In this work, we investigate the dynamics of charge generation and recombination in PIPCP:PC ${ }_{61} \mathrm{BM}$ blends. ${ }^{19,20}$ OSCs based on the donor-acceptor pairing of PIPCP and $\mathrm{PC}_{61} \mathrm{BM}$ (Figure 1c-e) have demonstrated one of the lowest energy losses reported $\left(E_{\text {loss }}=0.52 \mathrm{eV}\right)$ while maintaining a high external quantum efficiency $\left(\eta_{\mathrm{EOE}}>60 \%\right)$-yielding a $\eta_{\text {PCE }}$ of $\sim 6 \% .{ }^{19,20}$ Examination of the blend nanostructure with high-resolution transmission electron microscopy reveals highly ordered domains. Sensitive measurements of blend absorption by photothermal deflection spectroscopy (PDS) fit Urbach energies $\left(E_{\mathrm{U}}\right)$ of $27 \mathrm{meV}$; this suggests a low degree of electronic disorder, a feature which we confirm by molecular dynamics simulations which point to minimal variations in the dihedral angles between adjancent rings, even in an amorphous 
phase of the polymer. Additionally, complementary photoluminescence and electroluminescence spectroscopy show that the energy difference between photogenerated singlet excitons $\left(E_{\text {opt }}\right)$ and $E_{\mathrm{CT}}$ is $\sim 50 \mathrm{meV}$. Consequently, the low energy loss is attributed to a low degree of electronic disorder and a high $E_{\mathrm{CT}}$.

This material system thus presents itself as a model system as it exhibits minimal energy loss during the formation of charges and a high charge mobility aided by the low degree of electronic disorder. We use transient absorption (TA) spectroscopy to observe the evolution of the spectral signatures for excited states between $10^{-13}$ and $10^{-3} \mathrm{~s}$ - this is a direct probe of their population over these time scales. We find that charge generation proceeds on an ultrafast time scale, likely the result of rapid hole delocalization along the polymer backbone. This is supported by temperature-dependent measurements for the field-effect hole mobility $\left(\mu_{\mathrm{FET}}\right)$ that exhibits disorder-free transport. The high local mobility, however, exacerbates nongeminate, bimolecular charge recombination that competes with charge extraction on the $1-10 \mathrm{~ns}$ time scale and results in the formation of nonradiative triplet excitons. Combining these results, we are able to individually assess the decay yields for singlet and triplet CT states, providing a high level of detail into the spin-dependent recombination channels that control charge-recombination-induced energy loss.

\section{RESULTS}

PIPCP:PC ${ }_{61} \mathrm{BM}(1: 2)$ blends were prepared according to the procedure presented in the literature for optimal device performance. ${ }^{19,20}$ Figure le shows the steady-state absorption spectra of neat PIPCP films and PIPCP:PC ${ }_{61} \mathrm{BM}$ blend films measured by photothermal deflection spectroscopy. ${ }^{20}$ The absorption spectra are similar for both films, with the blend showing a $60 \mathrm{meV}$ red-shifted absorption with respect to the neat film. Ran et al. have reported the photoluminescence and electroluminescence spectra for PIPCP films and PIPCP:PC ${ }_{61} \mathrm{BM}$ blend films. ${ }^{20}$ These complementary techniques suggest that the $E_{\mathrm{CT}}$ is nearly resonant with the singlet exciton $\left(S_{1}\right)$, in contrast to numerous other reports for OSC systems where $E_{\mathrm{CT}}$ is generally found to be hundreds of meV lower. $^{21,22}$ To confirm this relationship, we have performed time-dependent density functional theory (TD-DFT) calculations of the $S_{1}$ and $E_{\mathrm{CT}}$ excitation energies; the TD-DFT calculations were carried out with the long-range corrected $\omega \mathrm{B} 97 \mathrm{X}-\mathrm{D}$ functional, a methodology that has been demonstrated to provide reliable results for both local (Frenkelexciton-type) and charge transfer excitations in extended $\pi$ conjugated systems. ${ }^{23}$ We examined a large number of polymer-fullerene configurations, which were generated randomly by having the fullerene molecule located at many different positions around the polymer backbone (see Supporting Information). The calculations predict that $E_{\mathrm{CT}}$ is ca. $0.1 \mathrm{eV}$ below $E_{\mathrm{opt}}$. Such a high $E_{\mathrm{CT}}$ is consistent with the high $V_{\mathrm{OC}}$ observed in this system and suggests that $\mathrm{T}_{1}$ in the donor polymer is lower in energy than $E_{\mathrm{CT}} \cdot{ }^{24}$ Importantly, for most of the polymer-fullerene configurations, the lowest excitation is found to have mixed Frenkel-CT character, with the electron wave function delocalized over both the polymer backbone and fullerene; this feature is consistent with the red shift of the lowest optical absorption observed upon going from the pure polymer to the blend.

Time-resolved TA is used to observe the evolution of exciton and charge populations after photoexcitation. Singlet exciton signatures can be used to quantify dissociation time scales. From pristine PIPCP film measurements, a signature corresponding to the singlet comprises a photoinduced absorption (PIA) peak near $1500 \mathrm{~nm}$ (Figure 2a). The decay
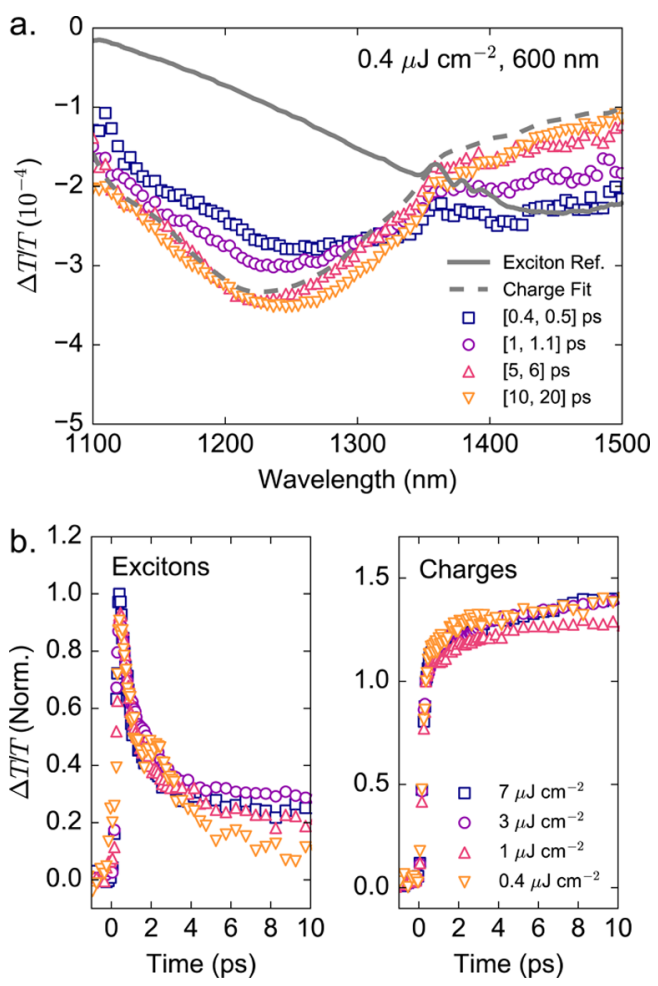

Figure 2. Efficient charge generation in the absence of excess energy. (a) Transient absorption spectra in the infrared for a PIPCP:PC ${ }_{61} \mathrm{BM}$ blend film for various times ranges between 0.4 and 20 ps measured at an excitation wavelength of $\lambda_{\text {ex }}=600 \mathrm{~nm}$ and excitation fluence of $0.4 \mu \mathrm{J} \mathrm{cm} \mathrm{cm}^{-2}$. (b) Kinetics for photogenerated excitons and charge carriers extracted from (a) as a function of excitation fluence.

of this feature in the blend film is attributed to exciton dissociation to form photoinduced charges (negative PIA feature at $1250 \mathrm{~nm}$ ). These two features are deconvoluted to extract their corresponding kinetics using a spectral deconvolution method based on a genetic algorithm described elsewhere. ${ }^{25}$ As shown in Figure $2 b$, most dissociation events are sub-picosecond, and all of them are completed within 10 ps. This process is independent of fluence between 0.4 and $7 \mu \mathrm{J}$ $\mathrm{cm}^{-2}$.

Figure 3 shows the TA for the blend film between 50 ps and $1 \mathrm{~ns}$. A clear isosbestic point is observed at $1220 \mathrm{~nm}$, indicating a population transfer from one state to another. This is explained by comparing the dynamics at 1100 and $1350 \mathrm{~nm}$ : the former decreases while the latter increases accordingly. This suggests that the $1100-1200 \mathrm{~nm}$ region is attributed mainly to charge PIA, whereas the $1250-1450 \mathrm{~nm}$ region is attributed mainly to triplet exciton PIA. Such would be the case if ${ }^{3} \mathrm{CT}$ states transfer energy to lower-lying $T_{1}$ states located on the PIPCP polymer chain. ${ }^{13}$

If these signatures are indeed due to the bimolecular recombination of charges, there should be a clear excitation fluence dependence where higher charge densities result in more collisions and faster triplet formation. Since the raw kinetics include contributions from multiple excited states 

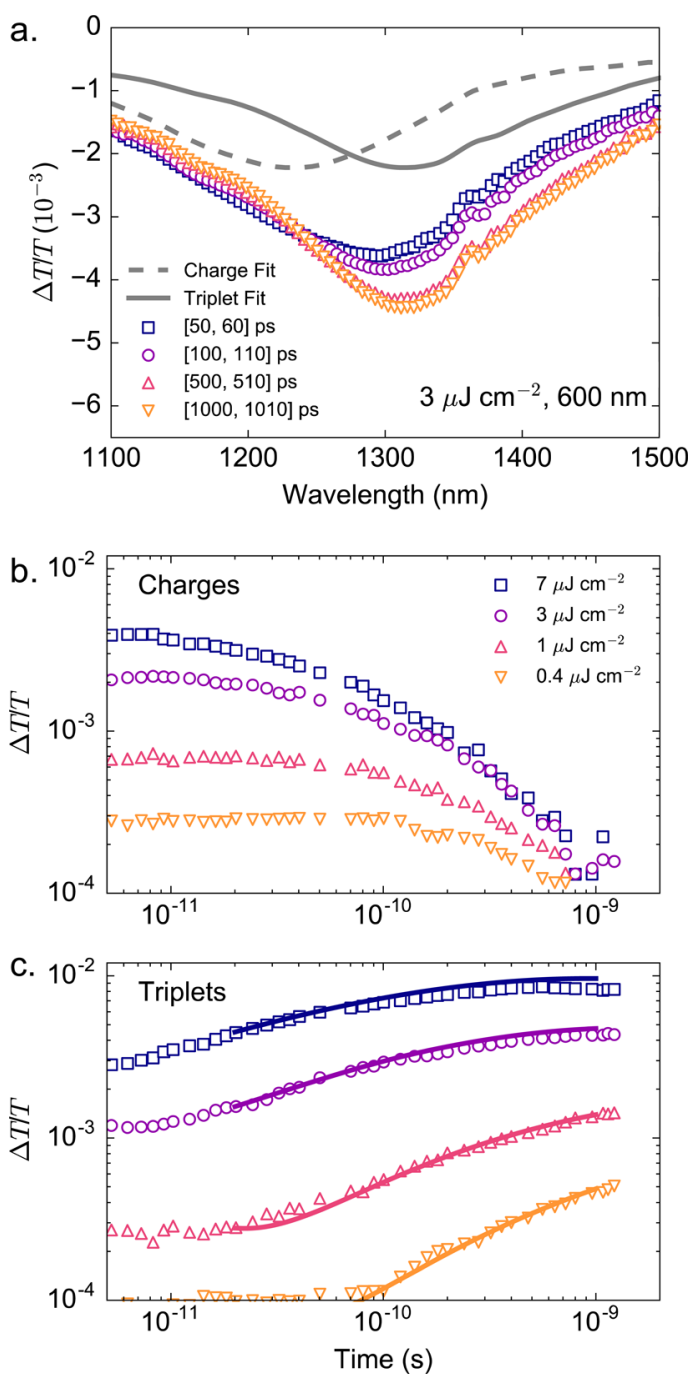

Figure 3. Fast bimolecular recombination of charges leads to the formation of nonradiative triplet excitons. (a) Transient absorption spectra for a PIPCP:PC ${ }_{61} \mathrm{BM}$ blend for various times ranging between $50 \mathrm{ps}$ and $1 \mathrm{~ns}$ measured at an excitation wavelength of $\lambda_{\mathrm{ex}}$ $=600 \mathrm{~nm}$ and excitation fluence of $3 \mu \mathrm{J} \mathrm{cm}^{-2}$. (b) Extracted kinetics for charges and (c) triplet excitons for a PIPCP:PC ${ }_{61} \mathrm{BM}$ blend as a function of fluence between 50 ps and $1 \mathrm{~ns}$.

overlapping at these wavelengths, an accurate description of the triplet dynamics requires proper extraction of the contribution from each species to the signal. We take advantage of the large amount of available data to numerically extract the underlying signatures and population for each species. ${ }^{26}$ The very clean data set and the presence of a well-defined isosbestic point makes this analysis unambiguous. By constraining the data extraction to solutions which are physically possible, optical signatures are rapidly obtained which are consistent across data sets of all fluences $\left(0.4-7 \mu \mathrm{J} \mathrm{cm}^{-2}\right)$ and time scales $\left(10^{-11}-\right.$ $10^{-6} \mathrm{~s}$ ) upon spectral deconvolution. The extracted spectral signatures are shown in Figure 3a. Figure $3 b, c$ shows the extracted charge and triplet kinetics between $10^{-11}$ and $10^{-9} \mathrm{~s}$. Charge and triplet kinetics from $10^{-9} \mathrm{~s}$ onward can be found in the Supporting Information (Figure S4). Confirmation of the triplet exciton PIA spectral signature is also found in the Supporting Information (Figure S5).

The population dynamics presented in Figure 3 show that triplet formation can happen on extremely fast time scales,
$10^{-11}-10^{-10} \mathrm{~s}{ }^{13,14}$ While fast triplet formation dynamics have been reported for a few other polymer-fullerene heterojunctions, the ones shown here for PIPCP:PC ${ }_{61} \mathrm{BM}$ blends demonstrate a sub-nanosecond formation time which is nearly 1 order of magnitude faster than other reports at equivalent excitation densities. ${ }^{13,14,27,28}$ For example, triplets are formed between $10^{-8}$ and $10^{-7} \mathrm{~s}$ for poly[2,6-(4,4-bis(2-ethylhexyl)$4 H$-cyclopenta[2,1-b;3,4-b9]-dithiophene)-alt-4,7-(2,1,3-benzothiadiazole)] (PCPDTBT) when processed without 1,8octanedithiol. ${ }^{14,27}$ When processed with the additive, the time scale for triplet formation shortens to $10^{-9}-10^{-8} \mathrm{~s}$, resulting from the increased mobility in this instance. ${ }^{14,29}$ In contrast to heterojunctions fabricated from PCPDTBT, however, the PIPCP:PC ${ }_{61} \mathrm{BM}$ blends studied do not exhibit geminate recombination. Kinetics describing the ground-state bleaching (GSB) do not decay over the time scales before the onset of bimolecular recombination, indicating that nongeminate, bimolecular recombination is the dominant recombination mechanism for PIPCP:PC ${ }_{61} \mathrm{BM}$ blends (Figure $4 \mathrm{a}$ ).
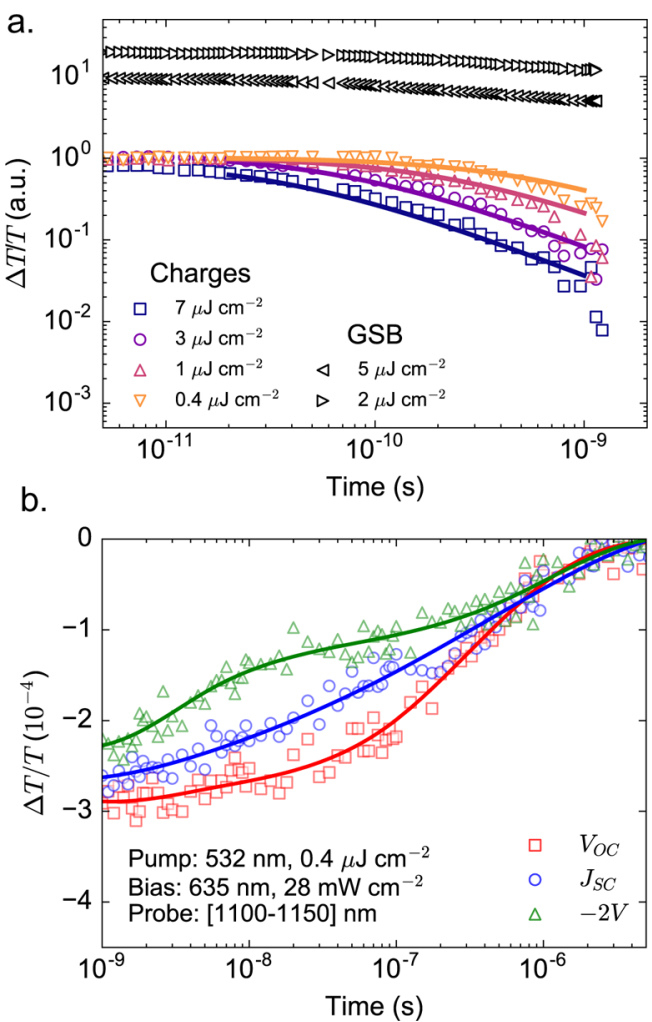

Figure 4. Competition between charge recombination and extraction. (a) Excitation intensity scaled charge kinetics for a PIPCP:PC ${ }_{61} \mathrm{BM}$ blend as a function of fluence between $50 \mathrm{ps}$ and 1 ns. Also shown are the kinetics of the ground-state bleach (GSB) offset by a factor of 10 and 20 for two fluences. (b) Polaron photoinduced absorption kinetics on a working PIPCP:PC ${ }_{61} B M$ device at various operating conditions.

The fast charge recombination and triplet formation suggests that charges are very mobile at early times and quickly collide nongeminately with others even at solar fluences. Moreover, the fast growth of the triplet population suggests that the coupling from the ${ }^{3} \mathrm{CT}$ state into the molecular triplet is fairly efficient despite the offset between the CT and triplet manifolds. ${ }^{30}$ To quantify the initial rate of charge recombina- 
tion, the charge kinetics in Figure $3 \mathrm{~b}$ are fit with a Langevin recombination model (Figure $4 \mathrm{a}):^{31}$

$$
\frac{\mathrm{d} N_{\mathrm{c}}}{\mathrm{d} t}=-k_{\mathrm{lan}} N_{\mathrm{c}}^{2}
$$

where $N_{\mathrm{c}}$ is the density of charges and $k_{\text {lan }}$ is the Langevin recombination rate constant defined as ${ }^{31}$

$$
k_{\text {lan }}=\frac{q\left(\mu_{\mathrm{h}}+\mu_{\mathrm{e}}\right)}{\varepsilon_{0} \varepsilon_{\mathrm{r}}}
$$

where $q$ is the charge of an electron, $\mu_{\mathrm{h}}$ and $\mu_{\mathrm{e}}$ are the hole and electron mobilities, $\varepsilon_{0}$ is the permittivity of vacuum, and $\varepsilon_{\mathrm{r}}$ is the relative dielectric constant. For $\varepsilon_{\mathrm{r}}=2$, the resulting mobility extracted from the kinetics in Figure $4 \mathrm{a}$ are $\mu_{\mathrm{h}}+\mu_{\mathrm{e}}=(5 \pm 0.3)$ $\times 10^{-3} \mathrm{~cm}^{2} \mathrm{~V}^{-1} \mathrm{~s}^{-1}$.

In order to establish a quantitative description for the triplet dynamics, we fit the entire set of triplet kinetics in Figure 3c according to the following equation used previously to describe the dynamics of charge and triplet populations in systems exhibiting triplet formation: ${ }^{13,14}$

$$
\frac{\mathrm{d} N_{\mathrm{T}}}{\mathrm{d} t}=-\alpha \frac{\mathrm{d} N_{\mathrm{c}}}{\mathrm{d} t}-\beta N_{\mathrm{T}} N_{\mathrm{c}}
$$

where $N_{\mathrm{T}}$ is the density of triplet excitons, $\alpha$ is the fraction of recombination events that leads to triplet formation, and $\beta$ is the triplet-charge annihilation rate constant. Importantly, the extracted charge kinetics are used as a direct input parameter for the fitting of the triplet kinetics, allowing for the specifics of charge recombination to be treated empirically. Good fits to the experimental data are achieved for $\alpha=(0.9 \pm 0.02)$ and $\beta=(7$ $\pm 0.2) \times 10^{-11} \mathrm{~cm}^{3} \mathrm{~s}^{-1}$.

Of note, the fraction of decay events that lead to triplet formation is $>0.75$, the value predicted according to the spin statistics for the recombination of two uncorrelated polarons. A system that exhibits $\alpha>0.75$ is still physically possible since CT states likely form and separate many times before decay ultimately occurs. ${ }^{6}$ This situation is supported by the concept of a reduced Langevin recombination factor $(\gamma)$ - a coefficient of the order $0.01-0.1$ needed to accurately describe charge recombination that occurs more slowly than what would be predicted solely from Langevin recombination. ${ }^{31}$ This point will be developed further in the discussion. The fit value for $\beta$ is on par with other reported values of $\beta$ in OSC blends. ${ }^{14}$

With these values determined, it is possible to estimate the contribution of this energy loss mechanism under short-circuit conditions. As the peak $\eta_{\mathrm{EQE}}$ for the PIPCP: $\mathrm{PC}_{61} \mathrm{BM}$ OSCs is $\sim 60 \%$, a conservative estimate for the internal quantum efficiency $\left(\eta_{\mathrm{IQE}}\right)$ is also $\sim 60 \%$ where the remaining $40 \%$ of charges are lost to premature recombination. As geminate recombination is negligible, we estimate that $36 \%$ of the uncollected charges under short-circuit conditions will be lost due to triplet formation. In contrast, only $4 \%$ are lost via direct recombination of ${ }^{1} \mathrm{CT}$ states. Note that the fast time scale for bimolecular recombination and triplet formation implies that these processes would compete with charge extraction when the device is operating at short-circuit and that, consequently, charges must also be collected on a similar time scale.

In order to confirm that charges are able to be extracted and contribute to photocurrent on the sub-10 ns time scale, charge extraction TA measurements were carried out by monitoring the polaron PIA signal in the infrared spectral region (1100$1150 \mathrm{~nm}$ ) on a working PIPCP:PC ${ }_{61} \mathrm{BM}$ device. Importantly, a continuous-wave laser operating at $\lambda=635 \mathrm{~nm}$ and $28 \mathrm{~mW}$ $\mathrm{cm}^{-2}$ was used to provide a background carrier density similar to that experienced at 1 sun illumination. Figure $4 \mathrm{~b}$ shows the kinetics of the charge population at $V_{\mathrm{OC}}$, short-circuit $\left(J_{\mathrm{SC}}\right)$, and $-2 \mathrm{~V}$ reverse bias. We observe that the population of charges in the device is dependent on the applied bias at times out to $\sim 100 \mathrm{~ns}$, with a strong suppression of the charge population as the bias condition is reduced from $V_{\mathrm{OC}}$ to short-circuit condition and finally to $-2 \mathrm{~V}$, due to rapid extraction of charges through the external circuit. However, there is residual charge population that is much longer lived, indicating that a fraction of the charge population, as much as $30 \%$, is much less mobile and much less suspectible to rapid extraction from the device. The largest difference in charge population occurs between 1 and $10 \mathrm{~ns}$, showing that the carriers that contribute to photocurrent are indeed those extracted at the earliest time scales-establishing that charge extraction competes with bimolecular recombination even in OSC heterojunctions with high mobility.

It should be noted that the $30 \%$ of residual trapped carriers cannot be directly compared with the approximate $40 \%$ missing from the $\eta_{\mathrm{EQE}}$ of the device. Under steady-state conditions, this longer lived population will stabilize and the probability that a charge becomes trapped will reduce. As bimolecular charge recombination and decay still compete with charge extraction on shorter time scales, the reduction in $\eta_{\mathrm{EQE}}$ can still be well rationalized.

As presented hereto, the degree of energetic and electronic order seen in PIPCP has been invoked to explain the presence of picosecond charge generation and sub-nanosecond charge decay and triplet formation. As will be shown, both of these mechanisms can be understood as the result of a high local mobility of hole polarons along the polymer backbone. We measured the hole $\mu_{\mathrm{FET}}$ for PIPCP and PIPCP:PC ${ }_{61} \mathrm{BM}$ blend films between 300 and $200 \mathrm{~K}$ (Figure 5). Temperature-

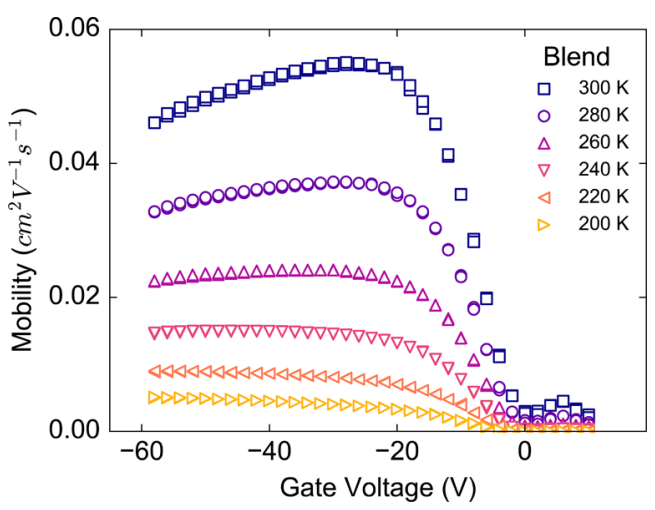

Figure 5. High, disorder-free local mobility for hole charge carriers. Field-effect mobility as a function of gate voltage at various temperatures between 200 and $300 \mathrm{~K}$ for a PIPCP:PC ${ }_{61} \mathrm{BM}$ blend film.

dependent measurements of $\mu_{\mathrm{FET}}$ for pure PIPCP films can be found in the Supporting Information. Of note, the hole mobility in the blend film shows a plateau in mobility upon increasing gate voltage. This behavior is similar to another indacenodithiophene (IDT)-based copolymer, IDT-BT, which also shows a large hole mobility of $\sim 2 \mathrm{~cm}^{2} \mathrm{~V}^{-1} \mathrm{~s}^{-1}$ and small $E_{\mathrm{U}}{ }^{32}$ This behavior has been attributed to nearly trap-free transport for holes aided by the rigidity of the IDT-based polymer backbone. In contrast to IDT-BT, however, the 
maximum $\mu_{\mathrm{FET}}$ measured for PIPCP:PC ${ }_{61} \mathrm{BM}$ blends is $\mu_{\mathrm{FET}}=$ $(0.05 \pm 0.01) \mathrm{cm}^{2} \mathrm{~V}^{-1} \mathrm{~s}^{-1}$. This lower value may reflect a smaller interchain coupling caused by a larger interchain separation due to the twisted phenyl side groups and signals the likelihood of dispersive transport. This is further evidenced by the voltage-independent tail in Figure $4 b$, which signals that some carriers may be trapped within the heterojunction with no accessible pathways for extraction, even under a reverse bias of $-2 \mathrm{~V}$. Nevertheless, this is the highest field-effect mobility yet reported for a donor-acceptor blend system, and it is higher than the threshold estimated for efficient PV device operation. $^{16-18}$

\section{DISCUSSION}

In the context of charge generation, a high local mobility for holes explains how charge generation can proceed so quickly. Unlike most high-efficiency OSC blends, where ultrafast charge generation is realized via rapid delocalization of an electron within a fullerene aggregate, ${ }^{26}$ PIPCP:PC ${ }_{61} \mathrm{BM}$ blends may instead rely on rapid delocalization of holes along the polymer backbone, underscoring the importance of polymer morphology in the generation of charges. ${ }^{33}$ This one-dimensional geometry of charge separation may also explain why the transient absorption of PIPCP:PC ${ }_{61} \mathrm{BM}$ blend films does not appear to exhibit electroabsorption as has been observed in other high $\eta_{\mathrm{PCE}}$ OSC heterojunctions (see Supporting Information). ${ }^{26}$

In the context of charge recombination, a high local mobility can also explain why the onset of bimolecular recombination is so fast. To further understand the interplay between the available recombination pathways in the blend, we can use the extracted information from the charge decay and triplet formation kinetics. Figure 6 shows a simple schematic

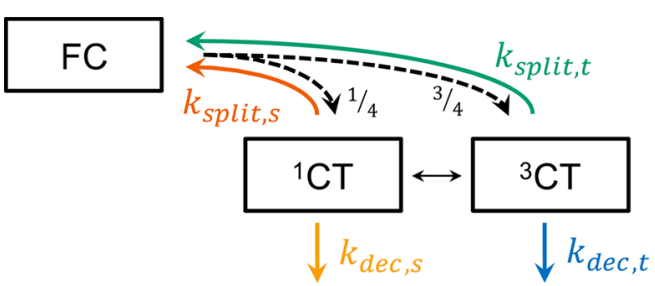

Figure 6. Spin-dependent charge recombination and decay pathways. Schematic diagram for a simplified scheme describing bimolecular recombination and spin-dependent charge transfer (CT) state decay within an organic heterojunction.

describing these pathways. Upon bimolecular recombination of free charges (FC), we expect from spin statistics that $25 \%$ will form ${ }^{1} \mathrm{CT}$ states and $75 \%$ will form ${ }^{3} \mathrm{CT}$ states. Once these states are formed, they will have individual rates to split $\left(k_{\text {split }}\right)$, re-forming free charges, or to decay to the ground state $\left(k_{\mathrm{dec}}\right)$. Note that intersystem and reverse intersystem crossings are also possible between the ${ }^{1} \mathrm{CT}$ and ${ }^{3} \mathrm{CT}$ states. In systems like PIPCP, however, where geminate recombination is negligible and CT states separate rapidly, the time scale for intersystem crossing is too slow to be competitive with $k_{\text {split }}$ and $k_{\text {dec }}{ }^{34}$

From the fitting of the triplet formation kinetics with eq 3 , we determined the total yield of recombination events that lead to triplet formation. Equation 4 shows how this yield is simply the result of successive encounters of the ${ }^{3} \mathrm{CT}$ state resulting from the recombination of FC. The yield can accordingly be written as an infinite geometric series where $\phi_{\mathrm{t}}$ is the probability that any given ${ }^{3} \mathrm{CT}$ will decay to $\mathrm{T}_{1}, \phi_{\mathrm{s}}$ is the probability that any given ${ }^{1} \mathrm{CT}$ will decay to $\mathrm{S}_{0}$, and $\gamma$ is the reduced Langevin recombination factor described earlier, providing the weighted probability of decay from both pathways. To be explicit, $\gamma$ is determined as the ratio between the Langevin rate constant experimentally extracted from fitting the charge decay kinetics in Figure $4 \mathrm{a}$ with eq $1\left(k_{\text {lan,exp }}\right)$ and that which can be predicted combining the measured $\mu_{\mathrm{FET}}$ and eq $2\left(k_{\text {lan,pred }}\right)$. The equations defining $\alpha, \gamma, \phi_{\mathrm{s}}$, and $\phi_{\mathrm{t}}$ are

$$
\begin{aligned}
& \alpha=\frac{3}{4} \phi_{\mathrm{t}} \sum_{i=0}^{\infty}(1-\gamma)^{i} \\
& \gamma=\frac{3}{4} \phi_{\mathrm{t}}+\frac{1}{4} \phi_{\mathrm{s}}=\frac{k_{\text {lan,exp }}}{k_{\text {lan, pred }}} \\
& \phi_{\mathrm{s}}=\frac{k_{\mathrm{dec}, \mathrm{s}}}{k_{\mathrm{dec}, \mathrm{s}}+k_{\mathrm{split}, \mathrm{s}}}, \phi_{\mathrm{t}}=\frac{k_{\mathrm{dec}, \mathrm{t}}}{k_{\mathrm{dec}, \mathrm{t}}+k_{\mathrm{split}, \mathrm{t}}}
\end{aligned}
$$

It is worth noting that, in contrast to previous reports determining $\gamma$, we have selected the field-effect mobility to tabulate $k_{\text {lan,pred }}$ instead of the mobility extracted from other techniques such as measurement of the space-charge-limited current (SCLC). ${ }^{35}$ For reference, PIPCP has a reported SCLC mobility of $1.4 \times 10^{-4} \mathrm{~cm}^{2} \mathrm{~V}^{-1} \mathrm{~s}^{-1} \cdot{ }^{19}$ Based on our observations of (1) sub-nanosecond formation of triplet excitons and (2) sub-10 ns extraction of charges that contribute to photocurrent, we assert that the charges relevant for determining the bimolecular recombination rate are indeed those which can move with a high mobility as prepared and measured within a field-effect transistor. Other techniques, which may prepare carriers predominantly in trapped states, would consequently not reflect the charge mobility that contributes to bimolecular recombination in a working device. Rather, these measurements might reflect the trapped carriers which recombine in a different manner, over longer time scales, and independent of applied bias (Figure 4b). Using a mobility value which is too small would lead to a dramatic overestimation of the overall decay yield $(\gamma)$ and result in a misinterpretation of the individual singlet and triplet decay yields.

For PIPCP:PC ${ }_{61} \mathrm{BM}$ blends, we tabulate $\gamma=0.1 \pm 0.02$. Combined with the measured value for the triplet yield of $\alpha=$ $0.9 \pm 0.02$, we can estimate that $\phi_{\mathrm{s}}=0.04 \pm 0.03$ and $\phi_{\mathrm{t}}=0.12$ \pm 0.02 , indicating that ${ }^{3} \mathrm{CT}$ states are three times more likely to decay (to form lower energy triplet states on the donor) than ${ }^{1} \mathrm{CT}$ states (that decay to the ground state). We extend this methodology to other polymer-fullerene heterojunctions reported in the literature. Table 1 compares the recombination yields of ${ }^{1} \mathrm{CT}$ and ${ }^{3} \mathrm{CT}$ states for PCPDTBT:PC ${ }_{71} \mathrm{BM}$ blends prepared with and without the solvent additive 1,8octanedithiol with those extracted for PIPCP:PC ${ }_{61} B M$. Of note, the overall yield of triplet excitons is larger for PIPCP than for either of the PCPDTBT blends. For the PCPDTBT blend processed without the additive, there is an increased tendency to recombine via ${ }^{1} \mathrm{CT}$, which disappears when the blend is processed with the additive. This is an important observation as there is not a large change in the decay yield for ${ }^{3} \mathrm{CT}$ excitons yet the overall triplet yield is still increased. In contrast, the enhanced triplet exciton yield for PIPCP blends appears to be a direct consequence of an enhanced tendency to undergo ${ }^{3} \mathrm{CT}$ to $\mathrm{T}_{1}$ energy transfer. 
Table 1. Extracted Recombination Yields for Singlet and Triplet CT States ${ }^{a}$

\begin{tabular}{cccc} 
& & \multicolumn{2}{c}{ PCPDTBT:PC ${ }_{71} \mathrm{BM}$} \\
\cline { 3 - 4 }$\alpha$ & PIPCP:PC ${ }_{61} \mathrm{BM}$ & without ODT & with ODT \\
$\gamma$ & $0.90 \pm 0.02$ & $0.57^{b}$ & $0.76^{b}$ \\
& $0.10 \pm 0.02$ & $0.07^{c}$ & $0.03^{c}$ \\
$\phi_{\mathrm{s}}$ & $0.04 \pm 0.03$ & & \\
$\phi_{\mathrm{t}}$ & $0.12 \pm 0.02$ & 0.12 & 0.03 \\
& & 0.05 & 0.03
\end{tabular}

${ }^{a}$ Error bars for $\alpha$ were determined from the standard deviation of the least-squares fit. Propagation of errors was used to tabulate the error bars for $\gamma, \phi_{\mathrm{s}}$, and $\phi_{\mathrm{t}} \cdot{ }^{b}$ See ref $14 .{ }^{c}$ Determined using eq 5 where $k_{\text {lan,exp }}$ is taken from literature ${ }^{36}$ and $k_{\text {lan,pred }}$ is estimated from field-effect mobility measurements. ${ }^{29}$

In order to further improve energy loss in the PIPCP:PC ${ }_{61} \mathrm{BM}$ system, it is imperative to reduce the rate of energy transfer to the triplet exciton state. One way to achieve this might be through further morphological optimization. In other OSC donor polymers, such as poly(indacenodithiopheneco-phenanthro[9,10-b]quinoxaline) (PIDT-PhanQ) and PCPDTBT, the presence of fullerene pure phases has been shown to establish a kinetic control of recombination that favors CT separation over decay, extending the charge lifetimes and increasing the $V_{\mathrm{OC}} \cdot{ }^{13,37}$ As the blend morphology for PIPCP:PC ${ }_{61} \mathrm{BM}$ blends is already optimized for maximal power conversion efficiency, however, this may not be achievable within the current set of materials. Perhaps a more attractive option is to remove the opportunity for triplet formation by selecting polymer donors where the $\mathrm{T}_{1}$ is above that of the CT state. Formation of polymer triplet states would then allow for their energy to be recovered via downhill energy migration. We note that materials with small singlet-triplet exchange energies are rapidly being developed for use in organic light-emitting diodes. These achieve low exchange energies through part separation of the electron and hole in the lowest excited state. ${ }^{38}$ While barriers for implementation of these materials in an OSC include their large energy gaps, weak CT absorption bands, and low carrier mobilities, promising paths forward may be uncovered as our understanding of these materials develops.

With this description in place, we can now understand the limits for recombination in PIPCP:PC ${ }_{61} \mathrm{BM}$ and PCPDTBT:PC ${ }_{71} B M$ heterojunctions which can, in turn, be extended to all OSC heterojunctions. If we consider that the rates of splitting for the ${ }^{1} \mathrm{CT}$ and ${ }^{3} \mathrm{CT}$ states are on the same order of magnitude, it follows that the decay rates for these states must also be on the same order of magnitude, albeit $\sim 10$ times smaller than those for splitting. As the rates are comparable, removing the nonradiative component of only one of these pathways will have a marginal impact on the overall rate of CT state decay and, hence, the energy loss and $V_{\mathrm{OC}}$ will be minimally affected. In the limit of a highly radiative ${ }^{1} \mathrm{CT}$ state, one should only need to reduce the ${ }^{3} \mathrm{CT}$ state decay rate to at or below that of the ${ }^{1} \mathrm{CT}$ state, thus approaching the radiative limit for energy loss within the heterojunction. As decay from the ${ }^{1} \mathrm{CT}$ and ${ }^{3} \mathrm{CT}$ states is mediated by separate mechanisms, realizing OSC blends at the radiative limit remains an achievable target.

\section{CONCLUSIONS}

In conclusion, we have described the pathways for charge generation and recombination in the low energy loss OSC based on PIPCP and $\mathrm{PC}_{61} \mathrm{BM}$. The small degree of electronic disorder is correlated to a high hole mobility along the polymer backbone. A high local mobility enables efficient, picosecond charge generation even though the singlet exciton and the CT states are nearly isoenergetic. The high local mobility, however, also speeds up bimolecular recombination, which here is predominantly through the formation of triplet excitons. Possible ways to alleviate the additional energy loss due to recombination are suggested, and a quantitative description for the limits of spin-dependent recombination was presented. Provided that the energy loss for PIPCP or other OSC blends can be further reduced, it is likely that a $\eta_{\mathrm{PCE}}$ exceeding $15 \%$ remains an achievable target for future generations of OSCs.

\section{METHODS}

Materials. PIPCP $\left(M_{\mathrm{n}}=66 \mathrm{kDa}\right.$, PDI $\left.=1.6\right)$ polymer was synthesized as reported elsewhere. ${ }^{19} \mathrm{PC}_{61} \mathrm{BM}$ was used as obtained from Solenne B.V. Zinc oxide $(\mathrm{ZnO})$ synthesis was done as per previous reports. ${ }^{39,40}$ In brief, the zinc oxide precursor solution was prepared by dissolving equimolar ratios of zinc acetate dihydrate $\left(\mathrm{Zn}\left(\mathrm{CH}_{3} \mathrm{COO}\right)_{2} \cdot 2 \mathrm{H}_{2} \mathrm{O}\right.$ (Sigma-Aldrich $\left.99.9 \%\right)$ and monoethanol amine $\left(\mathrm{NH}_{2} \mathrm{CH}_{2} \mathrm{CH}_{2} \mathrm{OH}\right)$ in 2-methoxyethanol $\left(\mathrm{CH}_{3} \mathrm{OCH}_{2} \mathrm{CH}_{2} \mathrm{OH}\right.$, Sigma-Aldrich 99.8\%) at room temperature (RT) and stirred at RT overnight for hydrolysis in air. PIPCP:PC ${ }_{61} \mathrm{BM}(1: 2, \mathrm{w} / \mathrm{w})$ blend and pristine PIPCP solutions were prepared in a mixed solvent of chloroform and chlorobenzene $(6: 4, \mathrm{v} / \mathrm{v})$ into a $20 \mathrm{mg} / \mathrm{mL}$ concentration and left to stir overnight.

Thin Film and Device Fabrication. Quartz and ITO glass substrates were cleaned by sonication in acetone and isopropyl alcohol for $15 \mathrm{~min}$ successively, followed by oxygen plasma treatment for $60 \mathrm{~s}$. Thin films were spin-coated at $2000 \mathrm{rpm}$ for $60 \mathrm{~s}$ in a $\mathrm{N}_{2}$-filled glovebox. For device fabrication of the ITO/ZnO/PIPCP:PC ${ }_{61} \mathrm{BM} /$ $\mathrm{MoO}_{x} / \mathrm{Au}$ solar cell, a $30 \mathrm{~nm}$ thick layer of $\mathrm{ZnO}$ was deposited on the cleaned ITO substrate using the $\mathrm{ZnO}$ precursor solution at $3000 \mathrm{rpm}$ for $45 \mathrm{~s}$ in air followed by annealing up to $250{ }^{\circ} \mathrm{C}$ in air. The substrates were then transferred to a $\mathrm{N}_{2}$-filled glovebox, and the 2:1 blend solution of PIPCP:PC ${ }_{61} \mathrm{BM}$ was spin-coated at $2000 \mathrm{rpm}$ for $60 \mathrm{~s}$. Finally, a $10 \mathrm{~nm}$ thick layer of $\mathrm{MoO}_{x}$ and a $100 \mathrm{~nm}$ thick layer of $\mathrm{Au}$ were successively deposited by a vacuum thermal evaporation method using an 8 pixel mask. The active area of the device was $4.5 \mathrm{~mm}^{2}$.

PV Measurement. Current-voltage $(I-V)$ characteristics were measured under $100 \mathrm{~mW} \mathrm{~cm}^{-2}$ equivalent AM1.5G conditions using an ABET Sun2000 solar simulator calibrated to a silicon reference cell corrected for spectral mismatch. The dark and the light $I-V$ characteristics were measured using a Keithley 237 SMU.

Photothermal Deflection Spectroscopy. PDS technique was used for the absorption measurements. Films spun on quartz substrates were used for the measurements. PDS is a highly sensitive scatter-free surface-averaged absorption measurement technique capable of measuring absorbances down to $10^{-5}$. A detailed description about the PDS setup can be found in the work of Sadhanala et al. ${ }^{41}$

Transient Absorption Spectroscopy. Optical pump and probe beams were generated from the output of a Ti:sapphire amplifier system (Spectra-Physics Solstice) operating at $1 \mathrm{kHz}$. One portion was used to pump a TOPAS optical parametric amplifier (Light Conversion) to generate a narrow-band pump pulse centered at $\lambda_{\mathrm{ex}}$ $=600 \mathrm{~nm}$. Another portion of the amplifier output was used to pump a series of home-built noncollinear optical parametric amplifiers to generate a broad-band probe pulse. Pump pulses for nanosecondmicrosecond time scale measurements were generated from the second harmonic $\left(\lambda_{\text {ex }}=532 \mathrm{~nm}\right)$ from a $q$-switched $\mathrm{Nd}: \mathrm{YVO}_{4}$ laser. The probe pulse was further divided to create a reference beam in order to correct for fluctuations in beam intensity and reduce the signal-to-noise ratio. The probe and reference beams were dispersed in a spectrometer (Andor, Shamrock SR-303i) and detected using a pair of $16 \mathrm{bit}, 512$ pixel linear image sensors (Hamamatsu). The probe was delayed using a mechanical delay stage (Newport) for picosecondnanosecond time scale measurements and electrically delayed for 
nanosecond-microsecond time scale measurements. Every second pump pulse was omitted using a mechanical chopper. Data acquisition at $1 \mathrm{kHz}$ was enabled by a custom-built board from Stresing Entwicklunsbüro. The differential transmission was calculated after accumulating and averaging over approximately $1 \mathrm{~s}$.

Genetic Algorithm. We use numerical methods based on a genetic algorithm to deconvolute the overlapping spectral signatures of individual excited states and obtain their kinetics. The full details of this approach can be found elsewhere. ${ }^{25}$ To summarize, a large population of random spectra are generated and modified to form successive generations of offspring, using a survival of the fittest approach. The best spectra are returned as optimized solutions. For a given solution, the fitness is calculated as the inverse of the sum of the squared residual with a penalty added for nonphysical results. The parent spectra are selected using a tournament method with adaptive crossover. The offspring are generated using a Gaussian function mask of random parameters.

Field-Effect Mobility Measurements. Organic bottom-contact top-gate OFETs with $20 \mu \mathrm{m}$ channel length and $1 \mathrm{~mm}$ channel width were fabricated on glass substrates with photolithographically patterned $\mathrm{Ti} / \mathrm{Au}(10 / 30 \mathrm{~nm})$ electrodes. For patterning the electrodes, a double layer lift-off process in $\mathrm{N}$-methyl-2-pyrrolidone was used. On top of the as-cast films, a $500 \mathrm{~nm}$ thick layer of CYTOP dielectric was deposited by spin-coating. Devices were finished off by evaporating a $25 \mathrm{~nm}$ thick layer of $\mathrm{Al}$ through a shadow mask. Room temperature transistor transfer characteristics were recorded inside a $\mathrm{N}_{2}$ glovebox using an Agilent 4155B semiconductor parameter analyzer. All low-temperature electrical measurements shown in this work were performed in a vacuum inside a Desert Cryogenics lowtemperature probe station.

Density Functional Theory Calculations. All DFT calculations were carried out using the long-range corrected $\omega$ B97X-D functional with the $6-31 \mathrm{G}(\mathrm{d}, \mathrm{p})$ basis set. ${ }^{42}$ Excited-state energies were calculated at the TD-DFT level using the Tamm-Dancoff approximation and considering the ground-state geometries. The impact of the surrounding dielectric medium (electron polarization effects) on $E_{\mathrm{CT}}$ was taken into account by combining the DFT calculations with the polarizable continuum model (PCM). Furthermore, the rangeseparation parameter $\omega$ of the $\omega \mathrm{B} 97 \mathrm{XD}$ functional is optimally tuned in the presence of the dielectric medium; all excited-state calculations were carried out with the PCM-tuned $\omega$ value. ${ }^{43}$ All DFT calculations were performed with the Gaussian 09 package. ${ }^{44}$

\section{ASSOCIATED CONTENT}

\section{S Supporting Information}

The Supporting Information is available free of charge on the ACS Publications website at DOI: 10.1021/acsnano.6b06211.

TD-DFT calculations for $S_{1}$ and $E_{\mathrm{CT}}$ excitation energies; broad-band transient absorption spectra for PIPCP:PC ${ }_{61} \mathrm{BM}$ blend films; extracted charge and triplet kinetics from $1 \mathrm{~ns}$ to $1 \mu \mathrm{s}$; confirmation of triplet photoinduced absorption spectrum; output characteristics for the PIPCP:PC ${ }_{61} \mathrm{BM}$ blend films; temperaturedependent mobility measurements for pure PIPCP films (PDF)

\section{AUTHOR INFORMATION}

\section{Corresponding Author}

*E-mail: rhf10@cam.ac.uk.

\section{Author Contributions}

S.M.M. and H.L.S. carried out the transient absorption spectroscopy. M.W. synthesized PIPCP polymer. A.S. performed the device and film fabrication and PDS measurements. M.N. performed the temperature-dependent field-effect mobility measurements. S.A.A. and M.K.R. carried out the theoretical calculations. S.M.M. analyzed the data and prepared the manuscript. All authors contributed to the development of the analysis and evaluation of the manuscript.

\section{Notes}

The authors declare no competing financial interest.

\section{ACKNOWLEDGMENTS}

S.M.M., R.H.F., M.K.R., S.A.-A., and J.-L.B. acknowledge support from the KAUST Competitive Research Grant Program. M.K.R., S.A.-A., and J.-L.B. also acknowledge generous support of their work by KAUST and the Office of Naval Research Global (Award N629091512003); they thank the KAUST IT Research Computing Team and Supercomputing Laboratory for providing computational and storage resources. N.A.R., M.W., T.-Q.N., and G.C.B. acknowledge support from the Department of the Navy, Office of Naval Research (Award Nos. N00014-14-1-0580 and N00014-16-125200. A.S. would like to acknowledge the funding and support from the India-UK APEX project. H.L.S. acknowledges support from the Winton Programme for the Physics of Sustainability. M.N. and H.S. gratefully acknowledge financial support from the Engineering and Physical Sciences Research Council though a Programme Grant (EP/M005141/1).

\section{REFERENCES}

(1) Brédas, J.-L.; Norton, J. E.; Cornil, J.; Coropceanu, V. Molecular Understanding of Organic Solar Cells: The Challenges. Acc. Chem. Res. 2009, 42, 1691-1699.

(2) Clarke, T. M.; Durrant, J. R. Charge Photogeneration in Organic Solar Cells. Chem. Rev. 2010, 110, 6736-6767.

(3) Vandewal, K.; Tvingstedt, K.; Gadisa, A.; Inganäs, O.; Manca, J. V. On the Origin of the Open-Circuit Voltage of Polymer-fullerene Solar Cells. Nat. Mater. 2009, 8, 904-909.

(4) Li, W.; Hendriks, K. H.; Furlan, A.; Wienk, M. M.; Janssen, R. A. J. High Quantum Efficiencies in Polymer Solar Cells at Energy Losses below $0.6 \mathrm{eV}$. J. Am. Chem. Soc. 2015, 137, 2231-2234.

(5) Shockley, W.; Queisser, H. J. Detailed Balance Limit of Efficiency of P-N Junction Solar Cells. J. Appl. Phys. 1961, 32, 510.

(6) Burke, T. M.; Sweetnam, S.; Vandewal, K.; McGehee, M. D. Beyond Langevin Recombination: How Equilibrium Between Free Carriers and Charge Transfer States Determines the Open-Circuit Voltage of Organic Solar Cells. Adv. Energy Mater. 2015, 5, 1500123.

(7) Gao, K.; Li, L.; Lai, T.; Xiao, L.; Huang, Y.; Huang, F.; Peng, J.; Cao, Y.; Liu, F.; Russell, T. P.; Janssen, R. A. J.; Peng, X. Deep Absorbing Porphyrin Small Molecule for High-Performance Organic Solar Cells with Very Low Energy Losses. J. Am. Chem. Soc. 2015, 137, $7282-7285$

(8) Ma, T.; Jiang, K.; Chen, S.; Hu, H.; Lin, H.; Li, Z.; Zhao, J.; Liu, Y.; Chang, Y.-M.; Hsiao, C.-C.; Yan, H. Efficient Low-Bandgap Polymer Solar Cells with High Open-Circuit Voltage and Good Stability. Adv. Energy Mater. 2015, 5, 1501282.

(9) Li, Y.; Liu, X.; Wu, F.-P.; Zhou, Y.; Jiang, Z.-Q.; Song, B.; Xia, Y.; Zhang, Z.-G.; Gao, F.; Inganäs, O.; Li, Y.; Liao, L.-S. Non-Fullerene Acceptor with Low Energy Loss and High External Quantum Efficiency: Towards High Performance Polymer Solar Cells. J. Mater. Chem. A 2016, 4, 5890-5897.

(10) Kawashima, K.; Tamai, Y.; Ohkita, H.; Osaka, I.; Takimiya, K. High-Efficiency Polymer Solar Cells with Small Photon Energy Loss. Nat. Commun. 2015, 6, 10085.

(11) Wang, C.; Xu, X.; Zhang, W.; Bergqvist, J.; Xia, Y.; Meng, X.; Bini, K.; Ma, W.; Yartsev, A.; Vandewal, K.; Andersson, M. R.; Inganäs, O.; Fahlman, M.; Wang, E. Low Band Gap Polymer Solar Cells With Minimal Voltage Losses. Adv. Energy Mater. 2016, 6, 1600148.

(12) Liu, J.; Chen, S.; Qian, D.; Gautam, B.; Yang, G.; Zhao, J.; Bergqvist, J.; Zhang, F.; Ma, W.; Ade, H.; Inganäs, O.; Gundogdu, K.; Gao, F.; Yan, H. Fast Charge Separation in a Non-Fullerene Organic Solar Cell with a Small Driving Force. Nat. Energy 2016, 1, 16089. 
(13) Rao, A.; Chow, P. C. Y.; Gélinas, S.; Schlenker, C. W.; Li, C.-Z.; Yip, H.-L.; Jen, A. K. Y.; Ginger, D. S.; Friend, R. H. The Role of Spin in the Kinetic Control of Recombination in Organic Photovoltaics. Nature 2013, 500, 435-439.

(14) Chow, P. C. Y.; Gélinas, S.; Rao, A.; Friend, R. H. Quantitative Bimolecular Recombination in Organic Photovoltaics through Triplet Exciton Formation. J. Am. Chem. Soc. 2014, 136, 3424-3429.

(15) Chen, X.-K.; Ravva, M. K.; Li, H.; Ryno, S. M.; Brédas, J.-L. Effect of Molecular Packing and Charge Delocalization on the Nonradiative Recombination of Charge-Transfer States in Organic Solar Cells. Adv. Energy Mater. 2016, 1601325.

(16) Tress, W.; Leo, K.; Riede, M. Optimum Mobility, Contact Properties, and Open-Circuit Voltage of Organic Solar Cells: A DriftDiffusion Simulation Study. Phys. Rev. B: Condens. Matter Mater. Phys. 2012, 85, 155201.

(17) Bartelt, J. A.; Lam, D.; Burke, T. M.; Sweetnam, S. M.; McGehee, M. D. Charge-Carrier Mobility Requirements for Bulk Heterojunction Solar Cells with High Fill Factor and External Quantum Efficiency > 90\%. Adv. Energy Mater. 2015, 5, 1500577.

(18) Bartesaghi, D.; Pérez, I.; Kniepert, J.; Roland, S.; Turbiez, M.; Neher, D.; Koster, L. J. A. Competition between Recombination and Extraction of Free Charges Determines the Fill Factor of Organic Solar Cells. Nat. Commun. 2015, 6, 7083.

(19) Wang, M.; Wang, H.; Yokoyama, T.; Liu, X.; Huang, Y.; Zhang, Y.; Nguyen, T.-Q.; Aramaki, S.; Bazan, G. C. High Open Circuit Voltage in Regioregular Narrow Band Gap Polymer Solar Cells. J. Am. Chem. Soc. 2014, 136, 12576-12579.

(20) Ran, N. A.; Love, J. A.; Takacs, C. J.; Sadhanala, A.; Beavers, J. K.; Collins, S. D.; Huang, Y.; Wang, M.; Friend, R. H.; Bazan, G. C.; Nguyen, T.-Q. Harvesting the Full Potential of Photons with Organic Solar Cells. Adv. Mater. 2016, 28, 1482-1488.

(21) Albrecht, S.; Vandewal, K.; Tumbleston, J. R.; Fischer, F. S. U.; Douglas, J. D.; Fréchet, J. M. J.; Ludwigs, S.; Ade, H.; Salleo, A.; Neher, D. On the Efficiency of Charge Transfer State Splitting in Polymer:Fullerene Solar Cells. Adv. Mater. 2014, 26, 2533-2539.

(22) Ran, N. A.; Kuik, M.; Love, J. A.; Proctor, C. M.; Nagao, I.; Bazan, G. C.; Nguyen, T.-Q. Understanding the Charge-Transfer State and Singlet Exciton Emission from Solution-Processed Small-Molecule Organic Solar Cells. Adv. Mater. 2014, 26, 7405-7412.

(23) Körzdörfer, T.; Brédas, J.-L. Organic Electronic Materials: Recent Advances in the DFT Description of the Ground and Excited States Using Tuned Range-Separated Hybrid Functionals. Acc. Chem. Res. 2014, 47, 3284-3291.

(24) Köhler, A.; Beljonne, D. The Singlet-Triplet Exchange Energy in Conjugated Polymers. Adv. Funct. Mater. 2004, 14, 11-18.

(25) Gélinas, S.; Paré-Labrosse, O.; Brosseau, C.-N.; Albert-Seifried, S.; McNeill, C. R.; Kirov, K. R.; Howard, I. A.; Leonelli, R.; Friend, R. H.; Silva, C. The Binding Energy of Charge-Transfer Excitons Localized at Polymeric Semiconductor Heterojunctions. J. Phys. Chem. C 2011, 115, 7114-7119.

(26) Gelinas, S.; Rao, A.; Kumar, A.; Smith, S. L.; Chin, A. W.; Clark, J.; van der Poll, T. S.; Bazan, G. C.; Friend, R. H. Ultrafast Long-Range Charge Separation in Organic Semiconductor Photovoltaic Diodes. Science 2014, 343, 512-516.

(27) Etzold, F.; Howard, I. A.; Forler, N.; Melnyk, A.; Andrienko, D.; Hansen, M. R.; Laquai, F. Sub-Ns Triplet State Formation by NonGeminate Recombination in PSBTBT:PC ${ }_{70} \mathrm{BM}$ and PCPDTBT:PC ${ }_{60} \mathrm{BM}$ Organic Solar Cells. Energy Environ. Sci. 2015, $8,1511-1522$

(28) Gehrig, D. W.; Howard, I. A.; Laquai, F. Charge Carrier Generation Followed by Triplet State Formation, Annihilation, and Carrier Recreation in PBDTTT-C/PC ${ }_{60} \mathrm{BM}$ Photovoltaic Blends. J. Phys. Chem. C 2015, 119, 13509-13515.

(29) Hwang, I.-W.; Cho, S.; Kim, J. Y.; Lee, K.; Coates, N. E.; Moses, D.; Heeger, A. J. Carrier Generation and Transport in Bulk Heterojunction Films Processed with 1,8-Octanedithiol as a Processing Additive. J. Appl. Phys. 2008, 104, 033706.

(30) Bittner, E. R.; Lankevich, V.; Gélinas, S.; Rao, A.; Ginger, D. A.; Friend, R. H. How Disorder Controls the Kinetics of Triplet Charge
Recombination in Semiconducting Organic Polymer Photovoltaics. Phys. Chem. Chem. Phys. 2014, 16, 20321-20328.

(31) Lakhwani, G.; Rao, A.; Friend, R. H. Bimolecular Recombination in Organic Photovoltaics. Annu. Rev. Phys. Chem. 2014, 65, 557581.

(32) Venkateshvaran, D.; Nikolka, M.; Sadhanala, A.; Lemaur, V.; Zelazny, M.; Kepa, M.; Hurhangee, M.; Kronemeijer, A. J.; Pecunia, V.; Nasrallah, I.; Romanov, I.; Broch, K.; McCulloch, I.; Emin, D.; Olivier, Y.; Cornil, J.; Beljonne, D.; Sirringhaus, H. Approaching Disorder-Free Transport in High-Mobility Conjugated Polymers. Nature 2014, 515, 384-388.

(33) Herrmann, D.; Niesar, S.; Scharsich, C.; Köhler, A.; Stutzmann, M.; Riedle, E. Role of Structural Order and Excess Energy on Ultrafast Free Charge Generation in Hybrid Polythiophene/Si Photovoltaics Probed in Real Time by Near-Infrared Broadband Transient Absorption. J. Am. Chem. Soc. 2011, 133, 18220-18233.

(34) Friend, R. H.; Phillips, M.; Rao, A.; Wilson, M. W. B.; Li, Z.; McNeill, C. R. Excitons and Charges at Organic Semiconductor Heterojunctions. Faraday Discuss. 2012, 155, 339-348.

(35) Proctor, C. M.; Kim, C.; Neher, D.; Nguyen, T.-Q. Nongeminate Recombination and Charge Transport Limitations in Diketopyrrolopyrrole-Based Solution-Processed Small Molecule Solar Cells. Adv. Funct. Mater. 2013, 23, 3584-3594.

(36) Etzold, F.; Howard, I. A.; Forler, N.; Cho, D. M.; Meister, M.; Mangold, H.; Shu, J.; Hansen, M. R.; Müllen, K.; Laquai, F. The Effect of Solvent Additives on Morphology and Excited-State Dynamics in PCPDTBT:PCBM Photovoltaic Blends. J. Am. Chem. Soc. 2012, 134, 10569-10583.

(37) Schlenker, C. W.; Chen, K.-S.; Yip, H.-L.; Li, C.-Z.; Bradshaw, L. R.; Ochsenbein, S. T.; Ding, F.; Li, X. S.; Gamelin, D. R.; Jen, A. K.-Y.; Ginger, D. S. Polymer Triplet Energy Levels Need Not Limit Photocurrent Collection in Organic Solar Cells. J. Am. Chem. Soc. 2012, 134, 19661-19668.

(38) Uoyama, H.; Goushi, K.; Shizu, K.; Nomura, H.; Adachi, C. Highly Efficient Organic Light-Emitting Diodes from Delayed Fluorescence. Nature 2012, 492, 234-238.

(39) Jagadamma, L. K.; Abdelsamie, M.; El Labban, A.; Aresu, E.; Ngongang Ndjawa, G. O.; Anjum, D. H.; Cha, D.; Beaujuge, P. M.; Amassian, A. Efficient Inverted Bulk-Heterojunction Solar Cells from Low-Temperature Processing of Amorphous $\mathrm{ZnO}$ Buffer Layers. J. Mater. Chem. A 2014, 2, 13321.

(40) Kim, G.-H.; García de Arquer, F. P.; Yoon, Y. J.; Lan, X.; Liu, M.; Voznyy, O.; Yang, Z.; Fan, F.; Ip, A. H.; Kanjanaboos, P.; Hoogland, S.; Kim, J. Y.; Sargent, E. H. High-Efficiency Colloidal Quantum Dot Photovoltaics via Robust Self-Assembled Monolayers. Nano Lett. 2015, 15, 7691-7696.

(41) Sadhanala, A.; Kumar, A.; Pathak, S.; Rao, A.; Steiner, U.; Greenham, N. C.; Snaith, H. J.; Friend, R. H. Electroluminescence from Organometallic Lead Halide Perovskite-Conjugated Polymer Diodes. Adv. Electron. Mater. 2015, 1, 1500008.

(42) Lin, Y.-S.; Li, G.-D.; Mao, S.-P.; Chai, J.-D. Long-Range Corrected Hybrid Density Functionals with Improved Dispersion Corrections. J. Chem. Theory Comput. 2013, 9, 263-272.

(43) Zheng, Z.; Brédas, J.-L.; Coropceanu, V. Description of the Charge Transfer States at the Pentacene/ $\mathrm{C}_{60}$ Interface: Combining Range-Separated Hybrid Functionals with the Polarizable Continuum Model. J. Phys. Chem. Lett. 2016, 7, 2616-2621.

(44) Frisch, M. J.; Trucks, G. W.; Schlegel, H. B.; Scuseria, G. E.; Robb, M. A.; Cheeseman, J. R.; Scalmani, G.; Barone, V.; Mennucci, B.; Petersson, G. A.; Nakatsuji, H.; Caricato, M.; Li, X.; Hratchian, H. P.; Izmaylov, A. F.; Bloino, J.; Zheng, G.; Sonnenber, D. J.; et al. Gaussian 09; revision A.02; Gaussian, Inc.: Wallingford, CT, 2009. 